\title{
Análise do perfil epidemiológico dos casos de coqueluche no estado do Piauí no período de 2013 a 2018
}

\author{
Analysis of the epidemiological profile of pertussis cases in the state of Piauí from 2013 to 2018 \\ Análisis del perfil epidemiológico de los casos de tos ferina en el estado de Piauí de 2013 a 2018
}

Recebido: 19/12/2020 | Revisado: 27/12/2020 | Aceito: 10/02/2021 | Publicado: 20/02/2021

\author{
Jôslanne dos Santos Soares \\ ORCID: https://orcid.org/0000-0001-7183-5847 \\ Universidade Federal do Piauí, Brasil \\ E-mail: joslannesoares@hotmail.com \\ Larisse Carneiro da Frota Brito \\ ORCID: https://orcid.org/0000-0003-2935-4163 \\ Universidade Federal do Piauí, Brasil \\ E-mail: larissa_frota13@hotmail.com \\ Maísa Barros Coêlho \\ ORCID: https://orcid.org/0000-0001-7805-543X \\ Universidade Federal do Piauí, Brasil \\ E-mail: maisabcoelho@gmail.com \\ Renata Kelly Espindola da Costa \\ ORCID: https://orcid.org/000 0-0003-2190-3420 \\ Universidade Federal do Piauí, Brasil \\ E-mail: renatakelly98@gmail.com \\ Evaldo Hipólito de Oliveira \\ ORCID: https://orcid.org/0000-0003-4180-012X \\ Universidade Federal do Piauí, Brasil \\ E-mail: evaldohipolito@gmail.com
}

\begin{abstract}
Resumo
A coqueluche é uma doença aguda, transmissível, de distribuição universal. Compromete especificamente o aparelho respiratório (traqueia e brônquios) e se caracteriza por paroxismo de tosse seca. Esse estudo tem como objetivo descrever os aspectos epidemiológicos da coqueluche no estado do Piauí, Nordeste do Brasil, de 2013 a 2018. Os dados foram retirados dos relatórios clínicos do Sistema de Informação de Agravos de Notificação (SINAN). Realizou-se análises descritivas dos números de casos notificados de coqueluche, segundo a faixa etária, o sexo e município mais afetados, bem como a evolução clínica. De 2013 a 2018 foram notificados 688 casos confirmados de coqueluche no Piauí. A capital do estado foi que apresentou maior número de notificação com 617 casos. Segundo a faixa etária, o maior número de notificações é de crianças menores de 1 ano de idade com 328 casos, seguido por crianças de 1 aos 4 anos com 103 casos. Em relação ao sexo na maioria das notificações as mulheres são as mais afetadas. A coqueluche é uma doença que ocorre sob as formas endêmicas e epidêmicas, tem seu indicio aumentada durante os anos, mas que possuem vacinação no calendário do SUS em crianças para sua prevenção.
\end{abstract}

Palavras-chave: Coqueluche; Epidemiologia; Evolução clínica; Doença.

\begin{abstract}
Pertussis is an acute, transmissible disease of universal distribution. It specifically compromises the respiratory tract (trachea and bronchus) and is characterized by paroxysm of dry cough. This study aims to describe the epidemiological aspects of pertussis in the state of Piauí, Northeast Brazil, from 2013 to 2018. The data were taken from the clinical reports of the Notifiable Diseases Information System (SINAN). Descriptive analyses of the numbers of reported cases of pertussis were performed, according to age group, sex and municipality most affected, as well as clinical evolution. From 2013 to 2018, 688 confirmed cases of pertussis were reported in Piauí. The state capital presented the highest notification number of 617 cases. According to the age group, the largest number of notifications is for children under 1 year old with 328 cases, followed by children aged 1 to 4 years with 103 cases. Regarding gender in most notifications women are the most affected. Pertussis is a disease that occurs in endemic and epidemic forms, has increased its indications during the years but have vaccination in the SUS calendar in children for its prevention.
\end{abstract}

Keywords: Pertussis; Epidemiology; Clinical course; Disease. 


\begin{abstract}
Resumen
La tos ferina es una enfermedad aguda, transmisible, de distribución universal. Afecta específicamente al aparato respiratorio (tráquea y bronquio) y se caracteriza por paroxismo de tos seca. Este estudio tiene como objetivo describir los aspectos epidemiológicos de la tos ferina en el estado de Piauí, Nordeste de Brasil, de 2013 a 2018. Los datos fueron tomados de los informes clínicos del Sistema de Información de Enfermedades Notificables (SINAN). Se realizaron análisis descriptivos del número de casos notificados de tos ferina, según grupo de edad, sexo y municipio más afectado, así como evolución clínica. De 2013 a 2018 se han notificado 688 casos confirmados de tos ferina en Piauí. La capital del estado fue que presentó mayor número de notificación de 617 casos. Según el grupo de edad, el mayor número de notificaciones es para niños menores de 1 año con 328 casos, seguido de niños de 1 a 4 años con 103 casos. En cuanto al género en la mayoría de las mujeres Las notificaciones son los más afectados. La tos ferina es una enfermedad que se produce en las formas endémicas y epidémicas ha aumentado su toque largo de los años, sino que tenga la vacuna en el calendario SUS en los niños para prevenirlos.
\end{abstract}

Palabras clave: Tos ferina; Epidemiologia; Evolución clínica; Enfermedad.

\title{
1. Introdução
}

A coqueluche é uma doença infecciosa aguda, cujo agente etiológico é a Bordatella pertussis, que tem como único reservatório natural o homem (Nobre, et al., 2016), acomete o trato respiratório, os brônquios e a traqueia principalmente. (Mattoo; Cherry, 2005). Trata-se de uma doença de alta transmissibilidade, porém, imunoprevenível. No entanto, a imunidade dada pela vacina é não permanente, mas duradoura, decrescendo com o tempo, até que sua proteção chegue a se mostrar reduzida ou nenhuma. (Willemann, et al., 2014).

A doença acomete todas as faixas etárias, porém crianças podem desenvolver apneia, cianose, pneumonia, hipertensão pulmonar, insuficiência respiratória e convulsões. (Torres, et al., 2015; Lynfield \& Schaffner, 2014). A transmissão ocorre pelo contato direto com a pessoa infectada, por meio de gotículas da orofaringe eliminadas por espirro, ao tossir ou falar. Enquan to a transmissão por contato indireto, é incomum, quando se utiliza material contaminado, pois o agente não sobrevive fora do hospedeiro. (Leite, et al., 2018).

Os primeiros sintomas da doença são observados em média de cinco a dez dias após a infecção, tendo seu percurso de evolução em três fases. A primeira fase se caracteriza por uma fase catarral, que dura em torno de uma a duas semanas. A segunda fase também chamada de paroxística, caracteriza-se por crise com tosse súbita rápida ou curta. E a terceira fase, chamada de fase de convalescença, dura em torno de duas a seis semanas, podendo prolongar-se por até três meses. (Mattoo \& Cherry, 2005; Tozzi, et al., 2005).

Segundo Leite, et al (2018), no Piauí em 2012 foram registrados 22 casos da doença, em 2013 esse número subiu para 100 e em 2014 teve um aumento significativo com registro de 377 casos. No entanto em 2015 a Secretaria de Estado da Saúde notificou sete casos da doença no Sistema de Informação de Agravos de Notificação (SINAN), sendo cinco casos em Teresina e dois em Barras.

Diante disso, este trabalho objetiva avaliar o perfil epidemiológico da coqueluche no estado do Piaú, no período de 2013 a 2018, de forma a avaliar a faixa etária, o sexo e os munícipios mais afetados, bem como a evolução clínica de forma a servir como uma alerta para os órgãos de saúde, para prevenção da doença.

\section{Metodologia}

O presente estudo consistiu em uma pesquisa retrospectiva com análises quantitativas, descritivas e documentais (Pereira et al., 2018), referentes aos casos notificados de coqueluche, no período de 2013 a 2018, no estado do Piauí. Para tal, utilizou-se os dados secundários extraídos no banco de dados do Departamento de Informática do Sistema Único de Saúde (DATASUS), onde estão disponibilizados no Sistema de Informações de Agravos e Notificação (SINAN). 
Como fonte de informação, fez-se o uso de publicações pertencentes aos bancos de dados Literatura LatinoAmericana e do Caribe em Ciências da Saúde - Lilacs, Medical Literature Analysis and Retrieval System Online - MedLine e Scientific Eletrônica Library - Scielo.

Como critério de avaliação, considerou-se a faixa etária, sexo, município e a evolução do quadro clínico do paciente. A análise estatística e a elaboração dos gráficos e tabelas foram realizadas por intermédio do programa Microsoft Excel 2016.

\section{Resultados e Discussão}

A coqueluche é uma doença de notificação compulsória no Brasil. E como a infecção e a vacinação não conferem imunidade a longo prazo, pessoas com baixa imunidade, dentre eles adolescentes e adultos, podem adquirir infecções que podem ser mais leves e/ou assintomáticas, ao qual dificulta o diagnóstico e torna essas populações fonte de infecção para lactentes, crianças ainda não vacinadas ou com esquema vacinal incompleto. (Willemann, et al., 2014).

$\mathrm{Na}$ Tabela 1, podemos observar o número de casos confirmados de coqueluche, notificados no Sistema de Informação de Agravos de Notificação (SINAN), no Piauí, no período de 2013 a 2018. A capital do estado, apresentou o maior número de notificações, 492 casos, um número bem elevado quando comparados ao dos outros municípios do Piauí.

O predomínio da coqueluche na cidade de Teresina, por ser justificado, já que se trata de um grande centro urbano, com populações aglomeradas, o que proporciona condições favoráveis para a circulação do bacilo, auxiliando na transmissão e a difusão da doença, já que o contato direto da pessoa doente com pessoa suscetível ou por objetos recém contaminados são suficientes para a sua propagação. Além disso, pelo fato de ser uma doença de distribuição universal e sua incidência ser independente da raça, clima e situação geográfica (Elias, et al., 2009).

Tabela 1. Casos confirmados ano, segundo município de residência, no PI, no período de 2013-2018.

Município de residência
Água Branca
Altos
Amarante
Arraial
Avelino Lopes
Baixa Grande do Ribeiro
Barra D'Alcântara
Barras
Batalha
Bertolínia
Boa Hora
Bom Jesus
Brasileira
Campo Largo do Piauí
Campo Maior
Caridade do Piauí
Castelo do Piauí
Coivaras
Corrente
Cristino Castro
Demerval Lobão
Elesbão Veloso
Esperantina
Fartura do Piauí
Floresta do Piauí
Floriano
Francisco Ayres
Francisco Macedo

\begin{tabular}{|l|l}
\hline 2013 \\
- \\
- \\
- \\
- \\
- \\
- \\
2 \\
- \\
- \\
- \\
- \\
1 \\
- \\
1 \\
- \\
- \\
- \\
2 \\
- \\
- \\
- \\
- \\
- \\
- \\
- \\
- \\
- \\
- \\
-
\end{tabular}

\begin{tabular}{|c|c|c|c|c|c|}
\hline 2014 & 2015 & 2016 & 2017 & 2018 & Total \\
\hline 2 & 5 & - & - & - & 7 \\
\hline 1 & 1 & - & - & - & 3 \\
\hline 1 & - & - & - & - & 1 \\
\hline- & 1 & - & - & - & 1 \\
\hline 1 & - & - & - & - & 1 \\
\hline 1 & - & - & - & - & 1 \\
\hline 1 & - & - & - & - & 1 \\
\hline 2 & 2 & 1 & - & - & 7 \\
\hline 2 & - & - & - & - & 2 \\
\hline 1 & - & - & - & - & 1 \\
\hline - & 1 & - & - & - & 1 \\
\hline 1 & - & - & - & - & 1 \\
\hline 1 & - & - & - & - & 2 \\
\hline- & 1 & - & - & - & 1 \\
\hline 1 & 1 & - & - & - & 3 \\
\hline 2 & - & - & - & - & 2 \\
\hline 1 & - & - & - & - & 1 \\
\hline - & 1 & - & - & - & 1 \\
\hline - & - & - & - & - & 2 \\
\hline 1 & - & - & - & - & 1 \\
\hline - & 2 & 1 & 1 & - & 4 \\
\hline 1 & - & - & - & - & 1 \\
\hline 1 & 3 & 1 & - & - & 5 \\
\hline - & - & - & - & - & 1 \\
\hline - & - & - & - & - & 1 \\
\hline 1 & 5 & - & - & - & 8 \\
\hline- & - & 1 & - & - & 1 \\
\hline 1 & - & - & - & - & 1 \\
\hline
\end{tabular}




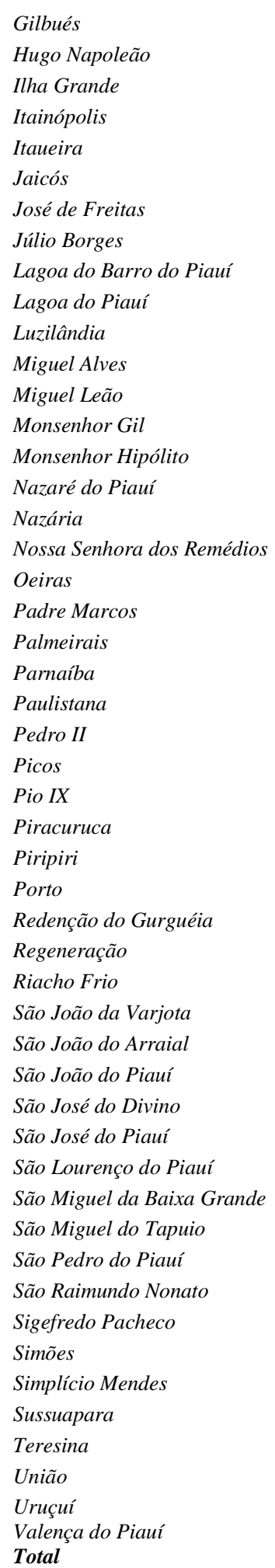

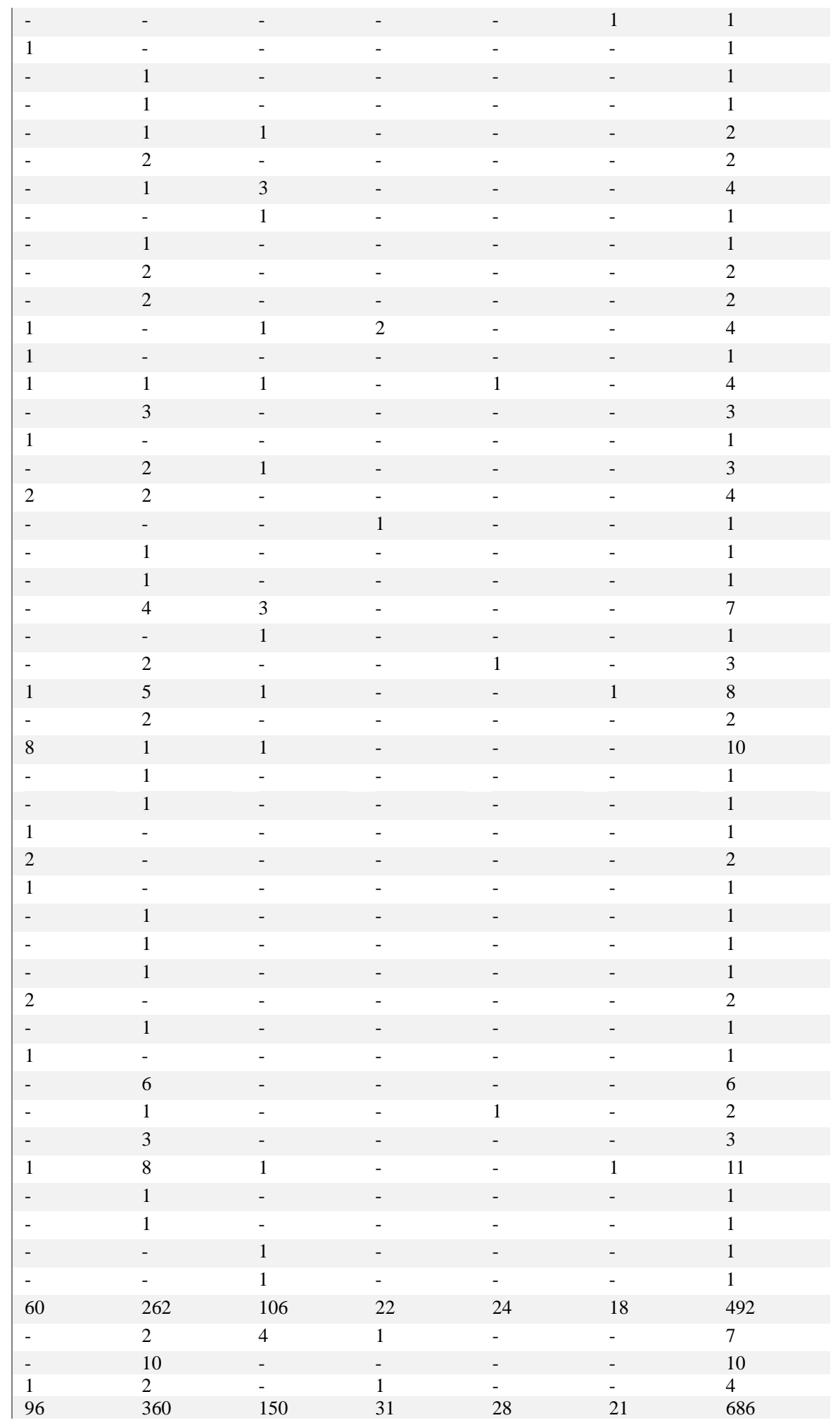

Fonte: Ministério da Saúde/SVS - Sistema de Informação de Agravos de Notificação - Sinan Net.

As crianças menores de um ano de idade, principalmente com menos de seis meses, são o grupo que apresentam taxas de letalidade e incidência mais acentuadas, a suscetibilidade desse grupo, pode ser decorrente de uma não vacinação contra a coqueluche ou devido a um esquema vacinal incompleto. (Trevizan \& Coutinho, 2008).

Sendo assim, no Piauí, a situação não é diferente e a faixa etária mais afetada é a de crianças menores de 1 ano de idade (343 casos), seguido por crianças de 1 a 4 anos (116 casos), como pode ser visto na Tabela 2 . Isso vai de acordo com 
grande parte dos estudos realizados, tais como o de Silva, Pereira e Martins (2015), nos quais, a prevalência de coqueluche ocorre também em menores de 1 ano de idade.

Tabela 2. Casos confirmados por ano, segundo a faixa etária, no PI, no período de 2013-2018.

\begin{tabular}{|c|c|c|c|c|c|c|c|c|c|}
\hline Ano & $<1$ Ano & $1-4$ & $5-9$ & $10-14$ & $15-19$ & $20-39$ & $40-59$ & $60 E+$ & Total \\
\hline 2013 & 53 & 20 & 7 & 1 & 7 & 8 & - & - & 96 \\
\hline 2014 & 157 & 70 & 51 & 32 & 2 & 33 & 11 & 4 & 360 \\
\hline 2015 & 88 & 17 & 15 & 5 & 5 & 17 & 1 & 2 & 150 \\
\hline 2016 & 14 & 4 & 1 & 1 & 2 & 6 & 2 & 1 & 31 \\
\hline 2017 & 21 & 1 & - & 2 & 1 & 3 & - & - & 28 \\
\hline 2018 & 10 & 4 & 1 & 6 & - & - & - & - & 21 \\
\hline Total & 343 & 116 & 75 & 47 & 17 & 67 & 14 & 7 & 686 \\
\hline
\end{tabular}

Fonte: Ministério da Saúde/SVS - Sistema de Informação de Agravos de Notificação - Sinan Net.

No Gráfico 1, observamos o número de casos confirmados com coqueluche por sexo ao longo do período de 2013 a 2018. A amostra foi constituída por 686 casos notificados, sendo o sexo feminino mais afetado no ano de 2014, com um número de 194 casos. No período de 2015 a 2018 houve um decréscimo significativo no número de casos confirmados com a doença, sendo o sexo feminino sempre o mais afetado. Porém a partir da análise do gráfico, segundo a literatura, pode-se confirmar que a bactéria $B$. pertussis não possui predileção por sexo, como observado no período em que houve o decréscimo. A diferença do número de casos notificados pode ser justificada devido ao processo de notificação da doença (Brasil, 2011). Segundo Pinto (2007), essa pequena diferença de notificação é devido a maior vigilância da doença em mulheres durante o período gestacional, por estas poderem transmitir a doença para os bebês, assim, aumentando os casos de coqueluche em recém-nascidos. (Pinto, 2007).

Gráfico 1. Caracterização dos casos de coqueluche notificados no SINAN, segundo o sexo, PI, no período de 2013 a 2018.

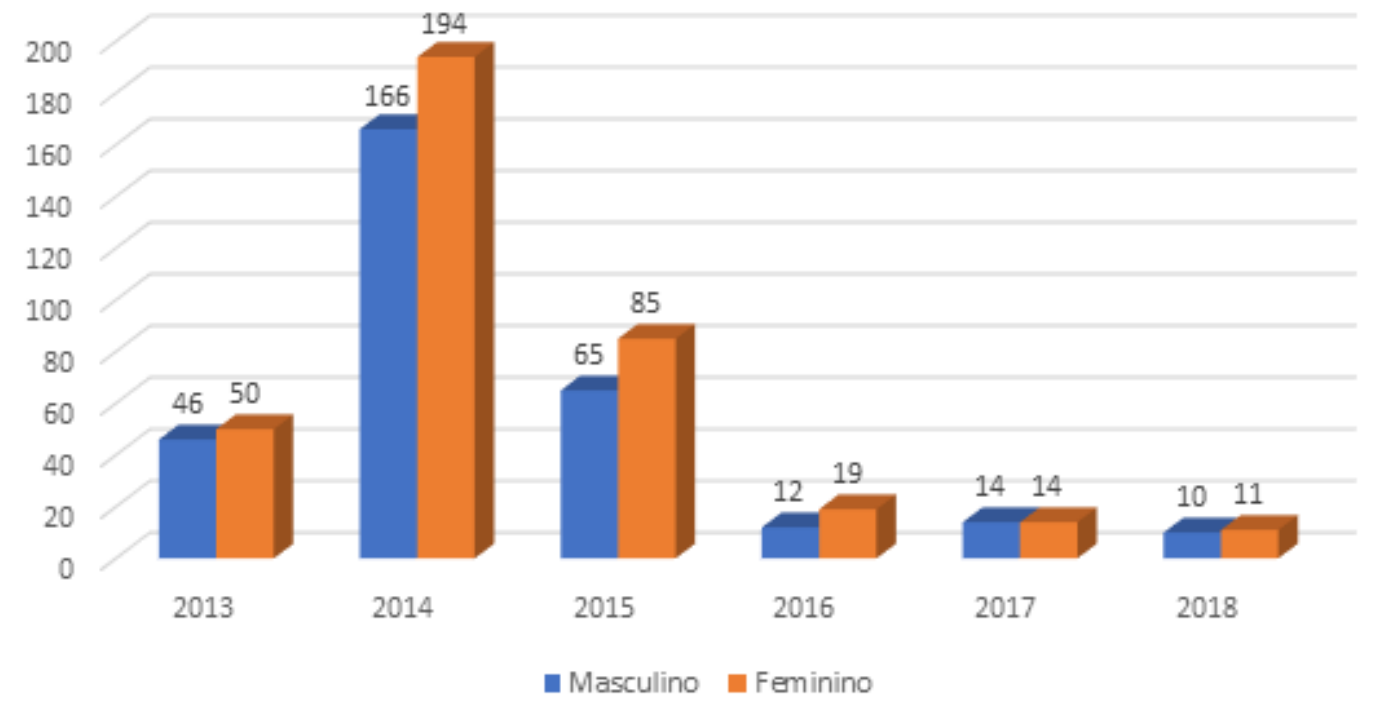

Fonte: Ministério da Saúde/SVS - Sistema de Informação de Agravos de Notificação - Sinan Net

A maioria dos casos de coqueluche possui uma boa resposta terapêutica e progridem para a cura. O protocolo de tratamento realizado nos pacientes é feito pela administração de antibióticos, como Azitrocimicina, Claritromicina, Eritromicina e SMZ-TMP, que são eficazes para a eliminação da bactéria na nasofaringe dos pacientes. O aumento significativo de pacientes evoluindo para a cura da doença, é justificado pelo advento de novos antibióticos. Entretanto, com o 
surgimento de uma cepa mais virulenta, essa poderia apresentar uma resistência aos antibióticos, gerando um aumento do número de casos da doença, como também aumentar a quantidade de casos que progridem para o óbito (Castro \& Milagres, 2017).

Com relação ao número de óbitos por coqueluche tem sido observado um aumento a partir de 2013 onde, 3 óbitos em crianças menores de 1 ano, pois confirma que estas são as mais gravemente acometidas pelo agravo devido seu fraco sistema imunológico e por não terem ainda o esquema completo de vacinação (Leite, 2019). Quanto a evolução 80,2\% evoluíram para a cura, com apenas 5 casos que foram a óbito (Gráfico 2), assim como pode ser observado que o maior índice de cura foi no ano de 2014. Além disso, no Gráfico 2, pode ser visto um expressivo número de campos nas fichas ignorado ou em branco, o que pode indicar que esteja ocorrendo uma subnotificação.

Gráfico 2. Caracterização dos casos de coqueluche notificados no SINAN, segundo a evolução dos casos, PI, no período de 2013 a 2018.

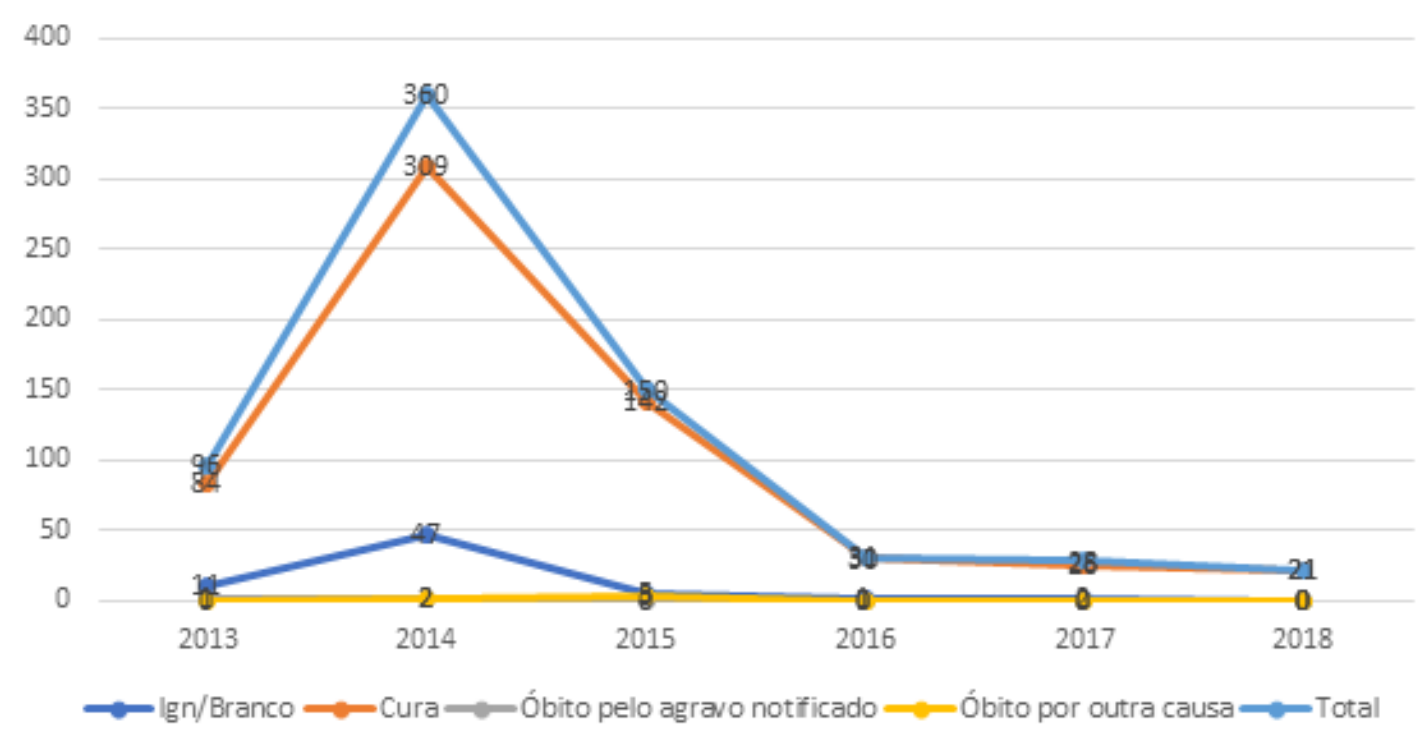

Fonte: Ministério da Saúde/SVS - Sistema de Informação de Agravos de Notificação - Sinan Net.

\section{Considerações Finais}

Não podemos afirmar que realmente esteja ocorrendo uma mudança no padrão epidemiológico da coqueluche no Estado do Piauí, uma vez que pode estar ocorrendo uma subnotificação da doença. Sendo necessário uma preparação dos profissionais para notificar corretamente. É necessária a realização de campanhas educacionais que conscientizem a população e os profissionais de saúde como um todo da importância das vacinações, visto que a maior faixa etária atingida está na fase da infância. Aquém isso, o poder público deve realizar medidas de fiscalização sanitária e deve garantir o acesso aos serviços de saúde a todos, em todas as regiões do Piauí, dando maior ênfase nas regiões que foram mais acometidas pela doença. Como perspectivas de trabalhos futuros pretendemos ampliar o estudo para todos os estados da região nordeste do Brasil.

\section{Referências}

Castro, H. W. V. D. \& Milagres, B. S. (2017). Perfil epidemiológico dos casos de coqueluche no Brasil nos anos de 2010 a 2014 . Universitas: Ciências da Saúde, Brasília, 15(2): 81-90.

Elias, C. D., Rocha, A. M., Werneck, L. D., Gomes, C. A., Brown, E., \& Monteiro, A. (2009). Caso fatal de coqueluche em um lactente. Pulmão RJ, 18(3), 155-157. 
Research, Society and Development, v. 10, n. 2, e39810211354, 2021

(CC BY 4.0) | ISSN 2525-3409 | DOI: http://dx.doi.org/10.33448/rsd-v10i2.11354

Leite, I. R. L., Guimarães, L. M., Rodrigues, A. C. E., Araújo, C. D. S. A., Silva, I. S. \& de Carvalho Neves, L. M. (2018). Perfil epidemiológico dos casos de coqueluche no Piauí no período de 2010 a 2015. Revista Ciência \& Saberes-Facema, 4(2), 1090-1096.

Lynfield, R. \& Schaffner, W. (2014). Can we conquer coqueluche? The Journal of infectious diseases, 209(suppl_1), S1-S3.

Mattoo, S. \& Cherry, JD (2005). Patogênese molecular, epidemiologia e manifestações clínicas de infecções respiratórias devido a Bordetella pertussis e outras subespécies de Bordetella. Clinical microbiology reviews, 18 (2), 326-382.

Nobre, J. O. C., Nunes, E. M., Yu, A. L. F., de Sousa, M. N. A., de Freitas, T. D., de Medeiros, R. C., \& Souza, F. S. (2017). Pertussis: Incidence and Surveillance in Municipalities of 6 Regional Health Management of Paraíba, Brazil. International Archives of Medicine, 9.

Pereira A.S. et al. (2018). Metodologia da pesquisa científica. UFSM. https://repositorio.ufsm.br/bitstream/handle/1/15824/Lic_Computacao_MetodologiaPesquisa-Cientifica.pdf?sequence $=1$.

Pinto, L. (2007). Imunização nos primeiros anos devida: as vacinas do programa nacional deimunização. Revista Hospital Universitário Pedro Ernesto, 6(1).

Silva, A. C., Pereira, J. L. \& Martins, T. C. (2015). Situação epidemiológica da coqueluche e dengue no Centro-Oeste e no Distrito Federal no período de 2001 a 2012. Trabalho de conclusão de curso (Bacharelado em Gestão em Saúde Coletiva). Universidade de Brasília, Brasília, 2015.

Torres, R. S., Santos, T. Z., Torres, R. A., Pereira, V. V., Fávero, L. A., Otavio Filho, R. M., \& Araujo, L. S. (2015). Resurgence of pertussis at the age of vaccination: clinical, epidemiological, and molecular aspects. Jornal de Pediatria (Versão em Português), 91(4), 333-338.

Tozzi, A. E., Celentano, L. P., Degli Atti, M. L. C., \& Salmaso, S. (2005). Diagnosis and management of pertussis. Cmaj, 172(4), 509-515.

Trevizan, S., \& Coutinho, S. E. D. (2008). Perfil epidemiológico da coqueluche no Rio Grande do Sul, Brasil: estudo da correlação entre incidência e cobertura vacinal. Cadernos de Saúde Pública, 24, 93-102.

Willemann, M. C. A., Goes, F. C. S., Araújo, A. C. M., \& Domingues, C. M. A. S. (2014). Adoecimento por coqueluche e número de doses administradas de vacinas Pertussis. Epidemiologia e Serviços de Saúde, 23, 207-214. 